Cahiers de géographie du Québec

\title{
Le sujet post-identitaire : nouvelles géographies sociales des campagnes ouest-africaines
}

\section{The Post-identity Subject: The New Social Geographies of Rural} West Africa

\section{El sujeto post-identitario: Nuevas geografías sociales de las regiones rurales del oeste africano}

\section{Angelo Turco}

Volume 54, numéro 153, 2010

URI : https://id.erudit.org/iderudit/1005600ar

DOI : https://doi.org/10.7202/1005600ar

Aller au sommaire du numéro

Éditeur(s)

Département de géographie de l’Université Laval

ISSN

0007-9766 (imprimé)

1708-8968 (numérique)

Découvrir la revue

Citer cet article

Turco, A. (2010). Le sujet post-identitaire : nouvelles géographies sociales des campagnes ouest-africaines. Cahiers de géographie du Québec, 54(153), 429-443. https://doi.org/10.7202/1005600ar

\section{Résumé de l'article}

De nouveaux enjeux se dessinent dans l'Afrique rurale subsaharienne, où le sujet vit de plus en plus son identité comme une construction complexe, au croisement de caractéristiques culturelles et de rôles sociaux hérités de la tradition avec les démarches aléatoires d'une conquête personnelle, dans des contextes géographiques en forte mutation. Les pouvoirs territoriaux se recomposent, entre autres, sur les bases de l'accès à l'espace et aux ressources naturelles et de la problématique de la responsabilité. L'exemple de la transformation des réseaux villageois est utilisé pour approfondir l'émergence de nouvelles identités. 


\section{Le sujet post-identitaire: nouvelles géographies sociales des campagnes ouest-africaines}

\author{
The Post-identity Subject: The New Social \\ Geographies of Rural West Africa \\ El sujeto post-identitario: Nuevas \\ geografías sociales de las regiones rurales \\ del oeste africano
}

\author{
Angelo TURCO \\ Université de l'Aquila \\ Turco@cc.univaq.it
}

\section{Résumé}

De nouveaux enjeux se dessinent dans l'Afrique rurale subsaharienne, où le sujet vit de plus en plus son identité comme une construction complexe, au croisement de caractéristiques culturelles et de rôles sociaux hérités de la tradition avec les démarches aléatoires d'une conquête personnelle, dans des contextes géographiques en forte mutation. Les pouvoirs territoriaux se recomposent, entre autres, sur les bases de l'accès à l'espace et aux ressources naturelles et de la problématique de la responsabilité. L'exemple de la transformation des réseaux villageois est utilisé pour approfondir l'émergence de nouvelles identités.

\section{Mots-clés}

Identité, formations sokunales, Afrique subsaharienne, campagnes.

\section{Abstract}

New challenges lie ahead in rural Sub-Saharan Africa, where, with increasing regularity, the subject perceives his/her identity as a complex construction, positioned at the crossroad of cultural features and social roles inherited from tradition and the random approach to individual conquest amid constantly evolving geographical contexts. Territorial power structures are redefined in terms of access to space and natural resources and the issue of accountability. A case in point depicts mutating village networks and their contribution toward a deeper understanding of emerging new identities.

\section{Keywords}

Identity, sokunal formations, Subsaharan Africa, rural areas. 


\section{Resumen}

Nuevas preocupaciones emergen en las regiones subsaharianas del África: el sujeto vive de más en más su identidad como una construcción compleja, a la interseción entre las características culturales y los roles sociales tradicionalmente heredados, incluyendo el progreso aleatorio de una conquista personal, en contextos geográficos en gran transformación. Los poderes territoriales se recomponen sobre fuentes de acceso al espacio y a las riquezas naturales, asi como sobre la problemática de la responsabilidad. El ejemplo de la transformación de las redes sociales aldeanas sirve para profundizar el delineamiento de las nuevas identidades.

\section{Palabras claves}

Identidad, formaciones sokunales, África subsahariana, regiones rurales.

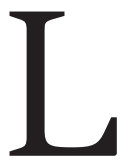

'Afrique change, de façon mimétique parfois et en suivant des trajectoires discontinues. Dans la grande variété des situations régionales, les communautés et les groupes sociaux se redéploient, les territorialités se reconfigurent. Depuis les indépendances, dont on fête en 2010 le cinquantenaire, les vieux clichés d'une terre figée dans ses traditions ont été bouleversés. Bien sûr, ces clichés restent largement utilisés par le système médiatique, mais cela n'empêche qu'ils sont à inscrire désormais dans la panoplie des idées reçues (Courade, 2006).

Pendant toute une première période - grosso modo durant la guerre froide -, les bouleversements majeurs se sont produits au niveau des mécanismes d'appropriation de la rente étatique et des circuits de l'économie moderne. Ces changements sont donc essentiellement urbains, avec quelques percées hors des villes, par l'agriculture de rente et le tourisme. Mais la chute du mur de Berlin et l'accélération du processus de mondialisation qui s'ensuit changent la donne car tout un mouvement de rénovation des rapports sociaux s'affirme non seulement dans les villes, mais aussi dans les campagnes. Les changements revêtent ici des caractères originaux, qui investissent aussi bien les plans économique que politique, notamment avec une mise en question des pouvoirs territoriaux.

La subjectivité est profondément concernée par ces bouleversements car, à côté des représentations qu'ils avaient d'eux-mêmes, les individus sont confrontés à de nouveaux mécanismes d'appartenance. Les liens ethniques et lignagers restent forts, bien entendu. L'empreinte religieuse, quant à elle, est bien vivante dans ses différentes articulations. L'origine régionale ne cesse d'alimenter des revendications aussi bien symboliques que matérielles. Mais à côté de tout cela, des nouveaux enjeux se dessinent, qui déterminent l'apparition d'un acteur social vivant de plus en plus l'identité non pas comme une condition dont on hérite, mais plutôt comme une conquête personnelle. Certains types de rapports, par exemple entre éleveurs et agriculteurs (Bonfiglioli, 1989; Bourgeot, 1999; Diallo et Schlee, 2000 ; Turner, 2004) ou entre immigrants et communautés d'accueil (Gaillard, 2000; Mounkaïla, 2002 et 2004 ; Turco, 2009a), suivent en partie seulement des schémas codifiés, et se déclinant de plus en plus en termes d'opportunités circonstancielles et de capacité de négociation des acteurs en présence. La consolidation de pratiques et d'institutions aussi variées que la coopération décentralisée, le multipartisme ou la décentralisation 
administrative, configure des "arènes locales » (Bierschenk et Olivier de Sardan, 1998; Olivier de Sardan, 1998 et 2006) où les anciens dépositaires de l'autorité villageoise doivent composer avec des gens capables d'appréhender rapidement les situations qui apparaissent à un moment ou à un autre, en se constituant en référence locale pour les interlocuteurs arrivant de l'étranger ou de la capitale. Il s'agit souvent d'anciens migrants, de jeunes ou de femmes, c'est vrai, mais peu importe puisque l'émergence de ces individus, jadis dépourvus en quelque sorte de subjectivité car catégorisés comme socialement peu importants, est susceptible d’apporter des bénéfices qui ne sont pas qu'individuels, car ils touchent à la collectivité tout entière (Schroeder, 1997 ; Dagobi, 2005, parmi bien d'autres).

Dans les espaces ruraux, désormais, l'identité n’est plus que socialement définie, surdéterminée en l'occurrence par des réseaux d'alliance, des obligations rituelles, des pratiques discursives. Elle ressemble moins à un ancrage solide, voire incontournable, qu'à une posture individuelle, flottante au gré des circonstances et des possibilités de négociation. Dans ces conditions, une véritable autoconstruction du soi apparaît, et de nouveaux rapports à l'espace s'inscrivent souvent parmi les expressions les plus marquantes de ce phénomène. Sur cette trame aux multiples facettes, quelques processus majeurs tirés d'expériences ouest-africaines sont présentés dans les pages qui suivent. Ils concernent notamment les problématiques de l'accès à la terre et aux ressources naturelles, de la responsabilité que les nouvelles figures d'autorité vont assumer dans les arènes locales et, finalement, des cadres évolutifs des réseaux villageois.

\section{L'accès et la responsabilité}

Depuis longtemps laboratoire social, la ville apparaît comme un moteur fondamental du changement. Elle reste notamment une plaque tournante qui assure la connexion entre les réalités nationales et le monde. Mais que se passe-t-il dans les campagnes? Comment les espaces ruraux réagissent-ils aux changements urbains, notamment quand il s'agit d'atténuer leur emprise hégémonique? En fait, on peut observer dans les campagnes ouest-africaines plusieurs dynamiques, liées à des facteurs tels qu'une nouvelle impulsion des cultures de rente (coton, noix d'acajou, cultures maraîchères), la dislocation des axes de transhumance due aux sécheresses sahéliennes, l'institution ou la revitalisation d'aires protégées et la décentralisation administrative. Sans oublier les «nouvelles guerres » qui ont profondément bouleversé l'organisation de l'espace de pays comme le Sierra Leone, le Liberia, la Côte d'Ivoire ou encore la Guinée-Bissau, et qui ont même impliqué de façon plus ou moins importante les espaces frontaliers. Sur un fond si complexe et mouvant, sillonné par des mouvements migratoires de toutes sortes, se réalise une véritable recomposition des pouvoirs territoriaux que nous allons illustrer par quelques exemples.

La recomposition des relations de pouvoir est fondamentale pour comprendre comment se définissent les nouvelles conditions d'accès à la terre et aux ressources naturelles. Dans leur ensemble, ces ressources constituent un facteur stratégique pour l'acquisition de nouvelles positions identitaires, mettant en jeu la capacité même de faire face aux défis de l'existence, à la jonction entre situation personnelle et architecture sociale. C'est ce que vise toute une nouvelle littérature scientifique sur l'accès, qui se penche sur cette «généalogie du pouvoir territorial» (Peluso et Watts, 2001), sans laquelle on risque de refaire ce qu'on a toujours fait: considérer 
l'État, ses organes périphériques et les institutions de la légalité en général comme les interlocuteurs uniques parce que sources premières et ultimes du droit foncier. En attendant qu'une philosophie du droit se formalise sur ce thème aussi flou que crucial - en reprenant par exemple les réflexions de la Geojurisprudenz du début du XIX ${ }^{\mathrm{e}}$ siècle (Irti, 2001) -, tout un débat s'inspire de ce legal pluralism qui a le mérite de mettre en lumière les graves responsabilités de l'État en matière foncière, à cause de son incapacité à discipliner juridiquement un domaine sur lequel il s'arroge pourtant la capacité de légiférer (Mathieu, 1996; Lavigne Delville, 1998; Leach et al., 1999; Michelot, 2005). Mais il apparaît indispensable d'intégrer aujourd'hui le domaine des droits de propriété - au sens du «droit positif» d'émanation étatique - et celui des droits d'usage au sens du droit "coutumier», avec une problématique des conditions d'acquisition de prérogatives concrètes sur l'espace et les ressources naturelles. L'accès se configure ainsi moins comme un droit que comme un enjeu, dans lequel on peut présumer l'identité comme une connotation de départ, tout en sachant que le sujet modifie son autoreprésentation par le biais du changement de la trame de ses rapports sociaux au cours du processus d'accession. Que l'on songe dans la pullangaku, la société fulbée, aux rimaybé, ces «peuls noirs» de condition servile qui, au contraire des rimbé, les "peuls rouges", ne peuvent être titulaires de droits fonciers mais seulement, et éventuellement, les exercer pour le compte de leurs maîtres. Que devient un "peul noir» projeté dans une dynamique d'accès, sinon quelqu'un qui vit dans une condition post-identitaire? Son succès éventuel le projette en effet bien au-delà du périmètre social que lui assigne le pulaaku, l'univers des valeurs peules (Leblon, 2006). Et ceci n'est pas sans effet dans la biographie du sujet, qui risque fort d'être exclu du pulaaku et d'être soumis au pulaagal, c'est-à-dire le code que les fulbés adoptent envers «ceux qui ne sont pas des peuls» (Monteil, 1950; Riesman, 1998).

Le processus d'accession permet donc à un sujet de jouir d'un bien (un champ, une jachère, un pâturage, un point d'eau, une forêt, une mare, un bas-fond, une mangrove, un marigot) qui ne lui est pas dû en fonction de ses marques identitaires - ethniques, professionnelles ou autres - mais sur lequel on a convenu quelque chose, par une démarche contractuelle, d'une façon plus ou moins consensuelle et plus ou moins durable. Dans le souci de systématiser la grande variété des situations empiriques personnelles, sociales, spatiales - Peluso et Ribot (2003) ont réfléchi à cette question de l'accès, entendu dans son sens le plus ample comme «la capacité de tirer avantage de l'espace et des ressources naturelles», au-delà du "droit de tirer avantage» qui fonde la propriété. Il y a là un déplacement capital de focalisation: d'un faisceau de prérogatives à un réseau de pouvoirs plus vaste et plus inclusif. Ceux-ci, quant à eux, s'exercent à trois niveaux qui se maintiennent rigoureusement séparés quoique étroitement interreliés. Le premier niveau est celui de l'acquisition de l'accès (gaining access), le premier stade à franchir pour participer au processus. Le deuxième est celui du contrôle de l'accès (access control), c'est-à-dire l'habilité à intervenir sur l'accès des autres. Il s'agit du pouvoir d'orienter et de réguler une action en principe libre, selon une acception très luhmanienne du pouvoir, non comme imposition mais comme conditionnement (Luhmann, 1982). Enfin, le troisième niveau est celui de la conservation de l'accès (access maintenance), qui consiste à maintenir ouvert l'accès à une ressource environnementale donnée.

On comprend comment les avantages, obtenus rapidement ou négociés au dernier moment en relation avec les acteurs, fonctionnent à différents niveaux. C'est comme si la position des acteurs par rapport au contrôle ou à la conservation de l'accès était 
constitutive des relations sociales portant sur l'appropriation, la gestion et l'utilisation des ressources environnementales. Tout cela montre encore une fois comment la gouvernance environnementale et le développement local sont les deux faces de la même médaille, en Afrique peut-être plus qu'ailleurs. Mais il faut aller plus loin dans la conception de l'analyse de l'accès comme ensemble de recherches permettant de comprendre quels sont les moyens, les relations et les processus à travers lesquels les acteurs sont en mesure d'acquérir, de contrôler et de conserver l'accès aux ressources environnementales. Ce que Peluso et Ribot appellent des «mécanismes »- et c'est un point capital - touche toujours et avant tout aux droits (right-based access). Et ceux-ci peuvent être licites ou illicites. Dans le premier cas, l'ambiguïté entre les systèmes d'autorité, qui joue un rôle de premier plan en Afrique, c'est-à-dire dans un contexte de pluralisme légal, est telle que l'on se réfère à différentes sphères normatives : légales et légitimes, comme nous les avons distinguées dans nos recherches (Turco, 2009a). Dans le second cas, il s'agit de pratiques malheureusement bien présentes en Afrique de l'Ouest, comme la violence, la corruption et le vol.

Mais les stratégies qui donnent forme aux processus d'accès produisent indirectement d'autres mécanismes ayant un cadre politique, économique et culturel plus général, dans lequel évoluent les acteurs. Toute une série de mécanismes d’accès structurels et relationnels entrent en jeu, fondés sur le mode avec lequel l'accès même subit l'influence de la technologie, du capital, du marché, de la connaissance et des relations sociales, sans oublier la cartographie (Peluso, 1995). Ceci n'est qu'une liste non exhaustive, bien entendu, à caractère principalement heuristique, qui nous dit toutefois l'ampleur de l'éventail des ressources complémentaires que les acteurs peuvent mobiliser dans des situations de conflit pour l'accès à l'espace et aux ressources naturelles.

La problématique de l'accès est étroitement liée à celle de la responsabilité (au sens de accountability, de rendre des comptes). De fait, il s'agit d'un thème délicat et même douloureux qui remonte à l'époque coloniale, quand ont été redéfinis les pouvoirs territoriaux sur la base des logiques hétérocentrées du colonialisme (Turco, 2000). Ce processus, a, en effet, conduit à la création de figures hybrides qui ont bouleversé les systèmes traditionnels de représentation: que l'on songe au chef de canton en Afrique de l'Ouest francophone, au regulo en domaine lusophone et à la panoplie de chiefs en domaine anglophone. Mais dans sa problématisation moderne, la responsabilité touche intimement à la décentralisation, que beaucoup considèrent comme une panacée, mais qui reste en réalité seulement une possibilité (Ribot, 1996, 1999 et 2005). Elle est certes importante mais, en elle-même, elle ne garantit rien. En particulier, elle ne garantit pas la démocratie locale et, à travers elle, le développement local, s'il ne se fait pas un transfert effectif de responsabilité sur le plan de la représentation politique. En d'autres termes, si celle-ci vient du bas (conférée aux élus par les électeurs), la responsabilité doit aller vers le bas et, donc, les élus doivent rendre des comptes aux électeurs. C'est facile à dire dans l'absolu, et cela semble même évident. Mais dans les faits, à nouveau, les choses ne se passent pas ainsi, puisqu'une mise en parallèle de la représentation et de la responsabilité introduit une innovation en matière de «gouvernementalité», pour reprendre l'expression célèbre de Michel Foucault, et bouleverse donc structurellement l'ensemble des relations entre les acteurs en présence.

D’un côté, en effet, accompagner le transfert de compétences pour assurer l'acquisition de responsabilité de la base signifie renoncer, au sein de la sphère de la légalité, à la part croissante d'autorité acquise par l'État et les systèmes de pouvoir qui s'y sont 
incrustés à des titres variés et selon des modes différents. Bref, c'est une remise en question de la rente étatique et des politiques du ventre qui s'y rattachent (Bayart, 1989). D’un autre côté, le nouveau contrat social exige que l'attention se focalise non seulement sur les nouveaux acteurs de la décentralisation, mais aussi sur une nouvelle base de légitimité, un authentique et inédit corps électoral (constituency). L’accent est mis, à nouveau, sur une dynamique post-identitaire, car tout cela souligne une connotation du sujet en tant qu'électeur, connotation dépassant toute une série de surdéterminations historiques, sociales et culturelles. Cela n'oblitère pas les vieilles appartenances et réseaux clientélistes, loin de là, mais induit de nouvelles perspectives d'action en ce qui concerne la compétition pour le pouvoir politique ou le contrôle des ressources économiques.

C'est pourquoi nous insistons depuis quelque temps sur le fait que, à propos de la soutenabilité, l'analyse actorielle est fondamentale pour comprendre ce qui se passe sur le terrain. Et ce, afin de répondre à des questions comme les suivantes : qui sont les interlocuteurs de la coopération au niveau local (Bierschenk et al., 2000) ? Comment et par quelles médiations s'exercent les pouvoirs territoriaux (Carvalho, 2003; Lund, 2006) ? Quel est le sens de l'expression "population locale» si nous introduisons l'idée d'une nouvelle base électorale (constituency) et si nous prenons en charge les nouvelles pratiques de légitimation (à partir des arguments développés par exemple par Mamdani, 1996)?

\section{Les formations sokunales}

Le mélange de ces nouveaux pouvoirs et des processus qu'ils alimentent directement ou indirectement, en particulier en Afrique rurale, a provoqué de nouvelles dynamiques au sein non seulement des villages, mais des structures intervillageoises. Comme on le sait, la territorialité villageoise - pas seulement ouest-africaine - se caractérise par un ensemble solide et fortement articulé de relations que le village développe avec l'espace environnant (qu'il est commode d'appeler «terroir», terme qui peut se révéler parfois ambigu) et avec un ensemble d'autres villages. Différentes par leur genèse, leurs contenus et leurs modalités de déroulement, ces relations ont des effets de proximité plus ou moins vastes et parfois hétéroclites, bien qu'étroitement mêlés. Mais nous voudrions nous pencher ici sur une configuration précise parmi les très nombreuses relations axées sur le village, qui n'a été que très peu étudiée par les géographes jusqu'ici mais qui s'avère particulièrement significative du point de vue que nous sommes en train de discuter. Cette configuration assume la forme générique du «village réticulaire», mais elle se traduit par une structure géographique tout à fait spécifique, car elle réalise l'inscription au sol d'un processus de légitimation territoriale basé sur la revendication par un village de «guider» les autres établissements humains. En Afrique de l'Ouest, c'est peut être la culture mandingue qui a travaillé à fond cette idée en la formalisant dans le concept de kunya - le fait, la prérogative, le droit de «prendre la tête». C'est ce qui nous pousse à appeler ce type de structure territoriale sokun, ce terme malinké (de so, village, et kun, tête) indiquant un réseau de villages, à savoir un regroupement de plusieurs implantations caractérisées par des rapports éminents avec un village-pivot (Turco, 2006). Il apparaîtra clair que généraliser ici une formation géographique par un terme malinké ne signifie en rien amoindrir les cultures locales, qui possèdent, elles aussi, des termes précis pour indiquer un village réticulaire sub specie de formation sokunale (Turco, 2009b). Il s'agit tout simplement 
d'une convention lexicale qui porte en elle une intention réflexive, comme le dirait Bourdieu (2001), à double facette. D’un côté, cette convention déclare comme peu pertinent le recours à une traduction en quelque langue véhiculaire, européenne ou autre, car les risques d'aliénation linguistique dénoncés chez les géographes par Raffestin (1981), à la suite des analyses de Rossi Landi (1968) et Calvet (1974), sont toujours présents. De l'autre côté, la convention lexicale invite - lorsqu'on s'engage dans des recherches plus approfondies sur l'argument - à appréhender les multiples stratifications sémantiques des différentes désignations en les replaçant dans leur contexte culturel et géographique particulier afin d'en saisir toutes les caractéristiques conceptuelles (parfois très fines) et les potentialités explicatives: ce sera alors pour le buiru (bariba) comme pour le diéma (gourmantché), le labu (djerma), le saaka (haoussa), le tamalhani (soussou), pour ne citer que les formations dont nous avons pu nous-mêmes approfondir l'étude.

En reprenant donc le fil rouge de notre argument, l'importance du sokun peut être perçue de différentes façons et à plusieurs niveaux. Dans les formations sokunales "classiques", le principe régulateur des rapports intervillageois est l'antériorité d'installation: la kunya, qui est perçue en même temps comme un pouvoir et une responsabilité, revient au village dont l'implantation est la plus ancienne. Le sokun touche donc aux sphères «classiques » de constitution de l'autorité en Afrique subsaharienne, ce trinôme famille/politique/sacré dont l'agencement social et spatial est tout à fait fondamental pour comprendre la dialectique des principes qui régissent la circulation légitime des pouvoirs. Or, les nouvelles dynamiques qui nourrissent l'émergence d'une subjectivité post-identitaire dans les campagnes ouest-africaines s'inscrivent en partie dans la tradition, quand elles concernent par exemple la dialectique des pouvoirs lignagers, politiques ou religieux au sens large. Pourtant, dans ce cas aussi, on remarque l'intervention de nouveaux protagonistes, porteurs de nouveaux intérêts, interprètes de nouvelles logiques dans l'exercice de l'autorité. Ces dynamiques génèrent des réalités tout à fait inédites et soutiennent la constitution de villages réticulaires qui perdent leurs connotations habituelles pour en acquérir de nouvelles. C'est ainsi qu'en Afrique de l'Ouest, à côté du sokun classique régi par des relations hiérarchiques fondées sur l'antériorité de l'établissement villageois, nous avons pu observer deux nouvelles formations réticulaires: le sokun écofonctionnel et le sokun flou.

Le sokun écofonctionnel peut être défini comme un ensemble de villages dont les relations naissent à partir d'une ressource naturelle commune, exploitée ou gérée par différents acteurs ; ce type de sokun peut tourner autour d'un ou de plusieurs villagespivots. Deux éléments doivent être pris en considération au sujet de la formation et du fonctionnement du sokun écofonctionnel. Le premier part de la constatation qu'un événement donné provoque la formation d'une «nouvelle» ressource naturelle. En d'autres termes, une «ancienne» ressource naturelle se transforme, sur les plans juridique, économique, territorial ou autre. L'événement auquel nous nous référons peut concerner par exemple un «projet» de coopération environnementale, ou bien, l'attribution par l'État d'une concession d'exploitation (minière, cynégétique, forestière), de grands travaux publics, d'aménagements hydrauliques ou autres (Bertoncin et Pase, 2008). 
C'est alors que deviennent cruciaux les intérêts qui, dans la nouvelle situation, se concentrent autour de la nouvelle ressource naturelle: quel est leur contenu objectif et calculable? Quelle est leur valeur symbolique? Quels en sont les acteurs et comment se situent-ils l'un par rapport à l'autre? Il n'est pas possible d'entrer ici dans le détail de ces enjeux, qui ont fait l'objet de réflexions multiples au cours de nos recherches. Il faut toutefois souligner que le sokun écofonctionnel peut certainement se former ex-novo autour de cette ressource, bien qu'il s'agisse plus souvent de villages-mères (tels qu'un soba malinké ou un doyuli gourmantché) et de villages-fils (soden; dobiga) déjà existants qui englobent des problématiques novatrices et finissent par devenir des sokun écofonctionnels. Cette métamorphose peut se produire dans des contextes de grave tension: c'est ce qui se passe lorsqu'il faut fuir, dans le cas d'un déplacement forcé des villages, d'une région qui devient aire protégée - ou zone tampon - vers des aires limitrophes, de la compétence autrefois d'autres villages du même sokun ou même d'autres sokun. Dans les cas de «déguerpissement» (expulsion), il va de soi que toutes les anciennes figures juridictionnelles sont bousculées et que l'organisation de l'implantation doit être entièrement redessinée suivant des critères qui ne dépendent pas d'une autorité mais d'un consensus. La métamorphose du sokun suit tout à fait les logiques de changement des pouvoirs et des ressources qui provoquent l'apparition de nouveaux acteurs, de nouveaux intérêts, de nouvelles stratégies territoriales, notamment dans des zones transfrontalières où interviennent aussi des rapports interétatiques.

La question de l'antériorité comme légitimant le pouvoir lignager présente toutefois deux aspects : d'un côté en effet, plusieurs sokun, c'est-à-dire plusieurs villages liés par des profils juridictionnels traditionnels, peuvent s'intéresser à une même ressource environnementale - une forêt, par exemple. Dans ce cas, un conflit de légitimité peut apparaître au sein du système lignager, en raison du changement de statut de la ressource en question. Car les anciens sokun permettaient de fixer les limites juridictionnelles des différents villages de manière adaptée à leurs attentes. L'intervention d'un acteur extérieur qui manifeste un intérêt conservateur pour la ressource forestière altère les équilibres antérieurs. Il n'est pas rare d'ailleurs que le projet considère comme unitaire un espace qui était fractionné autrefois - ou bien qu'il fractionne des espaces considérés autrefois comme unitaires - et qu'il introduise des critères rigides de délimitation dans des zones marquées autrefois par des frontières plutôt souples.

C'est ici qu'apparaît le deuxième aspect. Auparavant, chaque sokun pensait et agissait sur le plan territorial de façon autonome, suivant des intérêts, des perceptions et des capacités propres. Or, l'arrivée d'un nouvel acteur qui unifie les espaces des sokun, non seulement annihile cette autonomie, mais altère aussi profondément le caractère même de cette ressource. Car si sa gestion reposait auparavant sur les évaluations et le savoir de différents villages, par la suite, elle obéit à des réglementations techniques et juridiques complètement étrangères à la tradition précédente: la «compétence topique» des populations locales est remplacée par la «connaissance topique» des acteurs externes (Turco, 2005). En effet, le sujet qui possède une connaissance topique agit dans une logique de système dont relève la connaissance scientifique. En revanche, le sujet qui possède une compétence topique agit dans le cadre d'une logique de contexte; il ne connaît pas nécessairement la grammaire du système, mais il comprend l'intelligence de l'environnement, qu'il sait décoder et éventuellement recoder (Camara, 2008). De plus, alors que le rapport avec cette nouvelle ressource 
était conçu et pratiqué auparavant sur des bases non monétaires, dans la nouvelle organisation, les valeurs sont comptabilisées en termes de dépenses et de recettes, d'imputations et de transferts de sommes, qui peuvent en tout cas être facilement ramenés à un principe financier.

Faute de mieux, nous avons dénommé la deuxième innovation territoriale le sokun flou. Celui-ci se présente comme un ensemble de villages dont les liens, d'origines différentes, ont une force et une stabilité extrêmement variables, pouvant aller de la dépendance hiérarchique à des relations homologues; ces dernières subsument à leur tour des intérêts mutuellement reconnus qui évoluent en souplesse, dans un contexte de négociabilité. Il s’agit d'une définition qui esquisse évidemment un modèle de référence, mais qui se traduit sur le terrain par un développement très variable d'implantations et de rapports, marqués par des situations comme celles qu'illustrent par exemple, dans la périphérie béninoise du Parc W, plusieurs ensembles villageois multiethniques. Ici, plusieurs types de relations de pouvoir se recoupent, car ils arrivent à englober même une dizaine de villages et un nombre imprécis de campements, surtout guré peul, puisqu'ils sont situés sur l'axe de transhumance des troupeaux qui vont du Sahel nigérien vers les terres plus humides soudaniennes et guinéennes.

L'exemple que nous venons d'évoquer montre en réalité que des éléments flous s'introduisent dans toutes les formations sokunales, bien que de façon différenciée et parfois très nuancée. Cela tient aux innovations territoriales que nous allons illustrer par deux problématiques.

La première reprend la question du pastoralisme qui, au cours de ces dernières années, et surtout à la suite de la méridionalisation des transhumances, devient extrêmement complexe au sein du sokun. Car c'est ici que s'entremêlent des logiques qui, bien qu'elles soient toutes pastorales, diffèrent profondément suivant qu'elles concernent:

1) les éleveurs - ici on parle essentiellement des Peuls - installés de façon stable dans le sokun qui, à partir de leur guré, pratiquent d'habitude le yawtooru, c'est-à-dire une transhumance dans un rayon territorial étroit ; celle-ci peut se produire au sein du même espace sokunal, le long de parcours bien définis - wordugol, les couloirs de transit - qui mènent des différentes implantations (qu'il s’agisse de guré ou de villages) aux zones de pâturage ou aux points d'abreuvage;

2) les nouveaux venus qui, en attendant de décider s'ils s’installent définitivement ou s'ils vont se déplacer vers d'autres terres, pratiquent un élevage mixte - sédentaire et transhumant - à partir de leurs campements plus ou moins précaires ;

3) les éleveurs qui pratiquent la bartoje, la grande transhumance, et n'effectuent sur le terrain du sokun qu'un arrêt plus ou moins long, faisant donc temporairement pression sur les ressources locales en pâturage et en eau.

Cet univers pastoral tente de respecter les institutions des sokun, mais fonctionne en fait suivant des règles propres et repose sur un système d'autorité qui, dans les zones transfrontalières du Parc W par exemple (Burkina Faso, Bénin, Niger), se matérialise dans les figures du rugga et du garso. Ces deux figures incarnent la légitimité peule, 
surtout dans des contextes qui ne sont pas fulbé mais qui sont dominés par d'autres ethnies ou groupes socioprofessionnels; la première surtout constitue la référence suprême - politique et/ou lignagère - de la communauté peule, tandis que la seconde s'applique plus spécifiquement aux questions relatives à la transhumance et au pâturage. C'est à elles qu'on demande tout particulièrement de veiller au maintien de relations de coopération dans le sokun, au sein des institutions traditionnelles de régulation communautaire des alliances et des conflits.

La seconde problématique est liée à l'irruption de la «légalité» dans la société traditionnelle. Le pouvoir légal influence l'organisation du sokun de plusieurs façons, mais toujours à deux échelles principales. La première, l'échelle nationale, comprend le corpus législatif et réglementaire de l'État. Dans le monde rural, ce corpus se reflète surtout dans les lois cadres qui régulent l'accès à l'espace et aux ressources naturelles : le code foncier, le code forestier, le code pastoral. Ces lois présentent deux inconvénients majeurs, qui finissent par alimenter ensuite le caractère flou des sokun. Elles vont tout d'abord influencer des contextes dépourvus de références stables, comme le cadastre, où les droits et les limites dépendent des exigences de base, mais ne favorisent certainement pas l'application de normes calquées sur des modèles juridiques européens. Par ailleurs, ces différents codes constituent des encadrements de base qui requièrent, pour être un tant soit peu efficaces dans la pratique, des règlements d'application adéquats; dans la plupart des cas, ceux-ci sont absents et par conséquent la définition des tenants et des aboutissants, ou l'application de tel article, est laissée à l'interprétation - ou à «l'état d'âme» comme on dit dans les villages - d'un représentant quelconque de l'administration. C'est cette opacité qui conduit parfois à délivrer, sans une compétence effective, à des tiers étrangers aux sokun, des permis de chasse, de pêche ou même de coupe du bois, soit des droits sur des ressources qui font partie de la juridiction lignagère: avec les tensions et les conflits que l'on peut facilement imaginer.

Nous en arrivons à l'autre échelle d'action de la légalité, l'échelle locale, qui inclut différents organes de l'État, soient-ils administratifs (régions, préfectures, souspréfectures, secteurs...) ou techniques (services des forêts par exemple), émanant de ministères bien précis (celui des «Eaux et Forêts» est généralement le plus important). À ces figures très caractéristiques de l'administration s'ajoutent d'autres figures moins précises. Par exemple, celles chargées de faciliter la liaison entre le domaine de la légalité et celui de la légitimité dans les villages: les villageois utilisent souvent pour cette figure le terme générique «délégué». Ou encore celles qui doivent favoriser la gestion collective des ressources et des services, dans une perspective «légale» qui ignore - formellement du moins - les organisations «légitimes", lignagères ou autres. C'est ainsi que, dans les villages burkinabés, on trouve non seulement le RAV (Responsable administratif villageois, appelé précisément délégué), mais aussi toute une série de comités et de commissions dont les plus caractéristiques sont le CVGF (Comité villageois de gestion de la faune) et la CVGT (Commission villageoise de gestion des terroirs). Quant aux organes électifs liés à la décentralisation, tels les CRD (Communautés rurales de développement) en Guinée-Conakry, ils interviennent eux aussi pour alimenter le flou.

Le principe d'antériorité apparaît donc, pour des raisons multiples, de plus en plus incapable de réguler les différents types de rapports intervillageois, ce qui prélude à la crise de la structuration sokunale classique et à une réorganisation de l'équilibre 
des pouvoirs dans les campagnes. Les anciennes juxtapositions (ethniques et professionnelles notamment) ont du mal à rendre compte des réalités actuelles, tant au plan géographique que social. Les anciens codages qui scellaient la hiérarchie entre premier occupant et survenant (stable ou transhumant) sont modifiés au gré des situations par la qualité des intervenants extérieurs (administration, agents de la coopération, projets, investisseurs urbains, médiateurs de toute sorte), des enjeux dont il est chaque fois question, des pratiques, légales ou légitimes, qui peuvent au fur et à mesure s'exercer. Les ingrédients de la post-identité s'affirment par le biais des institutions élémentaires de type égalitaire qui tendent à accroître leur influence au fur et à mesure que les relations hiérarchiques s'affaiblissent. C'est ainsi que, dans les structures sokunales, les pouvoirs qui étaient autrefois confinés dans des rôles secondaires au sein des organisations traditionnelles deviennent de plus en plus visibles. Nous pouvons citer les classes d'âge, par exemple, ou les associations de femmes, les associations de métiers, de secours mutuel ou religieuses, aussi bien masculines que féminines. Ces formes associatives sont souvent à la base de la constitution d'ONG locales, qui participent volontiers à des réseaux plus vastes, gérés par une centrale urbaine.

C'est de cet univers bouillonnant, où les rôles sociaux se définissent de façon tâtonnante, que ressort une subjectivité post-identitaire au travers de ces nouvelles figures d'autorité s'affirmant sur la base de leur aptitude à déclencher et gérer des dynamiques socioterritoriales de type contractuel. D'autant plus que lorsque les intérêts de plusieurs sokun, classiques ou écofonctionnels, entrent en jeu, les relations qui s’instaurent sont nécessairement homologiques. Car dans ce cas, étant donné que plusieurs villages-mères réclament les mêmes privilèges dus à l'antériorité, les relations se nouent entre pairs et se réfèrent donc à des statuts, des intérêts et des prérogatives qui ne peuvent pas provenir d'une autorité préalable, ni se référer à des protocoles établis par la tradition, mais doivent être négociés, autour d'une «natte de concertation» par exemple. La conflictualité environnementale, qui endosse les connotations «modernes» de l'âge de la colonisation (Mackenzie, 1988; Pouchepadass, 1993 ; Calandra, 1999; McCann, 1999; Puyo, 2001), s'accroît tout en se fragmentant. Par conséquent, les dynamiques d'acteurs s'intensifient et les lieux de médiation se font de plus en plus nombreux, souvent en dehors des anciens cadres institutionnels de règlement des conflits (Calandra, 2008). Le flou prend la relève, les besoins de gouvernance s'affirment à côté des urgences de gouvernement.

\section{Vers où? Résiliences identitaires, intériorisation des normes, cadrages territoriaux}

Des nouveaux mouvements sociaux apparaissent en Afrique, dont la complexité ne peut nous échapper. D’autant plus qu'ils montrent des agencements territoriaux spécifiques qui replacent sur de nouvelles bases les rapports entre ville et campagne, d'un côté, et de l'autre, entre les différentes échelles d'action. Ces dynamismes sécrètent de nouvelles articulations de pouvoir qui, dans les campagnes notamment, mettent en question les fondements symboliques qui avaient, jusqu'à présent, assuré les équilibres entre les différents types d'autorité. Le paradoxe s'installe et un pouvoir étatique, de plus en plus puissant en apparence, se trouve dans l'obligation de composer localement avec ces nouvelles configurations de pouvoirs pour garantir le fonctionnement des 
dispositifs légaux. Par ailleurs, les autorités de base, relevant de la sphère lignagère aussi bien que politique et religieuse, doivent faire face aux changements de toute sorte qui font apparaître de nouveaux intérêts et de nouvelles solidarités mettant en question les fondements même de la légitimité ancestrale. Les figures traditionnelles de l'autorité doivent faire face à des remises en question continuelles et peuvent donc de moins en moins alimenter des processus d'identification et d'auto-identification. Par ailleurs, les campagnes ouest-africaines pullulent désormais de nouvelles figures socialement significatives, assurant par exemple la médiation politique entre échelle locale, échelle régionale et échelle nationale, ou bien la négociation sociale entre ville et campagne, ou encore le «courtage» en matière de coopération décentralisée.

L'Afrique rurale est-elle à l'heure du sujet post-identitaire? Plusieurs signes le montrent, d'autant plus que c'est bien plus qu'un repère chronologique qu'on évoque par cette expression, comme on l'a vu, car il s'agit de rendre compte d'un statut de la subjectivité dont le profil s'avère flexible. Certes, les résiliences identitaires sont multiples. L'intériorisation des nouvelles normes sociales induites par la post-identité est plutôt lente à se manifester clairement au plan politique. Les cadrages territoriaux ne reflètent qu'imparfaitement les nouvelles dynamiques de pouvoir. L’identité échappe progressivement aux formes cristallisées - qu'elles soient d'inspiration religieuse, lignagère ou politique - pour prendre l'aspect d'une fabrication de la personnalité où les discours et les pratiques s'entrecoupent, selon les contingences et les alliances possibles, tout en tenant compte des héritages historiques et culturels. Dans ce contexte, l'appartenance se reconfigure; elle n'est parfois qu'instrumentale, soumise aux aléas de la circonstance. Le projet de vie du sujet, avec ses chances de succès et ses risques d'échec, se couple aux suggestions mémorielles et aux conduites rituelles. Il n'est pas rare non plus, lors des processus migratoires par exemple, d'assister à l'adaptation des pratiques agraires des territoires d'attache aux territoires d'accueil. L'hybridation ne concerne pas seulement les techniques paysannes ou pastorales, mais aussi les conditions d'accès à la terre et aux ressources naturelles, la représentation politique, la responsabilité institutionnelle. La fluidité des situations confère une importance inédite, bien que problématique parfois, à des caractéristiques aussi bien individuelles que collectives telles que l'âge et le sexe. Dans ce même registre, nous pouvons remarquer que l'irruption de l'égalité comme valeur organisatrice investit la condition même de minorité sociale et engage dans des formidables processus d'autoconstrution de soi des sujets jadis enfermés dans des catégorisations discriminatoires: l'ancien esclave, le serviteur, le «non-né», l'homme (ou la femme) de caste, l'étranger.

Le sujet déchiffre continuellement sa propre généalogie, bien au-delà de ce qui lui a été raconté. Les rôles sociaux doivent composer désormais avec les biographies individuelles et familiales, à l'occasion d'événements marquants comme dans le vécu quotidien. L'entre-deux - la betweenness comme le dirait Entrikin (1991) - devient le grand chantier des identités nouvelles. Des géographies inédites se mettent en place, dont la recomposition sociale devient un signe marquant et, de plus en plus, une condition nécessaire. 


\section{Bibliographie}

BAYART, Jean-François (1989) L'État en Afrique: la politique du ventre. Paris, Fayard.

BERTONCIN, Marina et PASE, Andrea (2008) Attorno al Lago Ciad. Sguardi diversi sullo sviluppo. Turin, L'Harmattan Italia.

BIERSCHENK, Thomas et OLIVIER DE SARDAN, Jean-Pierre (dir.) (1998) Les pouvoirs au village. Le Bénin rural entre démocratisation et décentralisation. Paris, Karthala.

BIERSCHENK, Thomas, CHAUVEAU, JeanPierre et OLIVIER DE SARDAN, JeanPierre (2000) Courtiers en développement. Les villages africains en quête de projets. Paris, Karthala.

BONFIGLIOLI, M. Angelo (1989) Dudal. Histoire de famille et histoire de troupeau chez un groupe de Wodaabe du Niger. Paris, Maison des sciences de l'homme.

BOURDIEU, Pierre (2001) Science de la science et réflexivité. Paris, Raisons d'agir.

BOURGEOT, André (dir.) (1999) Horizons nomades en Afrique sahélienne: société, développement, démocratie. Paris, Karthala.

CALANDRA, Lina (1999) Politiche ambientali e colonialismo: l'Africa Occidentale Francese. Terra d'Africa, no VIII, p. 39-89.

CALANDRA, Lina (2008) Conflitti territoriali a Tamou (Niger): i poteri in questione. Storia Urbana, n 118 , p. 91-120.

CALVET, Louis-Jean (1974) Linguistique et colonialisme. Petit traité de glottophagie. Paris, Payot.

CAMARA, Laye (2008) Saperi basici e conservazione ambientale: i Malinké dell'Alto Niger (Guinea Conakry). Storia Urbana, $\mathrm{n}^{\circ} 118$, p. 41-68.

CARVALHO, Clara (2003) A revitalização do poder tradicional e os regulados manjaco da Guinée-Bissau. Soronda, Décembre, p. 7-44.

COURADE, Georges, dir. (2006) L'Afrique des idées reçues. Paris, Belin.
DAGOBI, A. Elhadji (2005) Les pouvoirs locaux et le rôle des femmes à Tamou. Niamey, LASDEL.

DIALLO, Youssouf et SCHLEE, Günther (dir.) (2000) L'ethnicité peule dans des contextes nouveaux. La dynamique des frontières. Paris, Khartala.

ENTRIKIN, J. Nicholas (1991) The betweenness of place: towards a geography of modernity. Baltimore, Johns Hopkins University Press.

GAILLARD, Gérald (dir.) (2000) Migrations anciennes et peuplement actuel des côtes guinéennes. Paris, L'Harmattan.

IRTI, Natalino (2001) Norme e luoghi. Bari, Laterza.

LAVIGNE DELVILLE, Philippe (dir.) (1998) Quelles politiques foncières pour l'Afrique rurale? Réconcilier pratiques, légalité et légitimité. Paris, Karthala.

LEACH, Melissa, MEARNS, Robert et SCOONES, Ian (1999) Environmental entitlements: dynamics and institutions in Community-Based Natural Resource Management. World Development, $\mathrm{n}^{\circ} 2$, p. 225-247.

LEBLON, Anaïs (2006) Le pulaaku. Bilan critique des études de l'identité peule en Afrique de l'Ouest. Aix-en-Provence, CEMAF.

LUHMANN, Niklas (1982) Potere e codice politico. Milan, Feltrinelli.

LUND, Christian (2006) Twilight institutions: public authority and local politics in Africa. Development and Change, $\mathrm{n}^{\circ} 4$, p. 685-705.

MACKENZIE, M. John (1988) The empire of nature. Hunting, conservation and british imperialism. Manchester, Manchester UP.

MAMDANI, Mahmood (1996) Citizen and subject. Contemporary Africa and the legacy of late colonialism. Princeton, Princeton University Press. 
MATHIEU, Paul (dir.) (1966) Démocratie, enjeux fonciers et pratiques locales en Afrique. L'Harmattan, Paris.

McCANN, James (1999) Green land, brown land, black land an environmental history of Africa 1800-1900. Portsmouth, Heinemann.

MICHELOT, Agnès (2005) Harmonisation des cadres institutionnels et législatifs nationaux. Conakry, Rapport AGIR.

MONTEIL, Charles (1950) Réflexions sur le problème des Peuls. Journal des Africanistes, no 2, p. 153-192.

MOUNKAÏLA, Harouna (2002) De la migration circulaire à l'abandon du territoire local dans le Zarmanganda (Niger). Revue européenne des migrations internationales, $\mathrm{n}^{\circ} 2$, p. 161-187.

MOUNKAÏLA, Harouna (2004) Migration, chefferie et accès aux ressources foncières dans le canton de Torodi (Ouest du Niger). Les Cahiers d'Outre-Mer, nos 226-227, p. 137-154.

OLIVIER DE SARDAN, Jean-Pierre (1998) Chefs et projets au village (Niger). Bulletin de l'APAD, no 15, p. 65-89.

OLIVIER DE SARDAN, Jean-Pierre (2006) Quelques réflexions autour de la décentralisation comme objet de recherche. Bulletin de l'APAD, no 16, p. 165-171.

PELUSO, Nancy L. (1995) Whose woods are these? Counter-mapping forest territories in Kalimantan, Indonesia. Antipode, vol. 27, nº 4, p. 383-406.

PELUSO Nancy L. et WATTS, Michael, (dir.) (2001) Violent environments. Ithaca, Cornell University Press.

PELUSO Nancy L. et RIBOT Jesse C. (2003) A theory of access. Rural Sociology, vol. 68, $\mathrm{n}^{\circ} 2$, p. 153-181.

POUCHEPADASS, Jacques (dir.) (1993) Colonisation et environnement. Paris, L'Harmattan.
PUYO, Pierre-Yves (2001) Sur le mythe colonial de l'inépuisabilité des ressources forestières (Afrique occidentale française/Afrique équatoriale française), 19001940). Cahiers de géographie du Québec, vol. 45, no 126, p. 479-496.

RAFFESTIN, Claude (1981) Pour une géographie du pouvoir. Paris, Litec.

RIBOT, Jesse C. (1996) Participation without representation: chiefs, councils and rural representation. Cultural Survival Quarterly, n³, p. 40-44.

RIBOT, Jesse C. (1999) Decentralization, participation and accountability in Sahelian forestry: legal instruments of politicaladministrative control. Africa, $\mathrm{n}^{0} 1, \mathrm{p}$. 23-65.

RIBOT, Jesse C. (2005) Building local democracy through natural resource interventions. An environmentalist's responsibility. Washington, World Resources Institute.

RIESMAN, Paul (1998) Freedom in fulani social life. Chicago, University of Chicago Press.

ROSSI LANDI, Ferruccio (1968) Il linguaggio come lavoro e come mercato. Una teoria della produzione e dell'alienazione linguistiche. Milano, Bompiani.

SCHROEDER, A. Richard (1997) Re-claiming land in The Gambia: gendered property rights and environmental intervention. Annals of the Association of American Geographers, vol. 87, no3, p. 457-508.

TURCO, Angelo (2000) Colonisation et après : légitimité territoriale et développement durable en Afrique subsaharienne. Dans Vincent Berdoulay et Olivier Soubeyran (dir.) Milieu, colonisation et développement durable. Perspectives géographiques sur l'aménagement. Paris, L'Harmattan, p. 175-184.

TURCO, Angelo (2005) Problématique du sujet et compétence topique: regards sur et depuis l'Afrique subsaharienne. Hégoa, no 25 , p. 57-63.

TURCO, Angelo (2006) Sokun. Le village réticulaire en Afrique de l'Ouest. Géographies et cultures, $\mathrm{n}^{\circ} 60$, p. 111-133. 
TURCO, Angelo (2009a) Governance, culture, sviluppo. Cooperazione ambientale in Africa Occidentale. Milan, Franco Angeli.

TURCO, Angelo (2009b) Paesaggio e discorso in Africa subsahariana: il caso dei Gurmancé (Burkina Faso, Niger, Benin, Togo, Gana). Dans A. Bagnasco et al., Le frontiere della geografia, Torino, UTET, p. 333-365.

TURNER, D. Mattew (2004), Political ecology and the moral dimensions of «Resource Conflicts»: the case of farmer-herder conflicts in the Sahel. Political Geography, no 7, p. 863-889. 